\title{
SHAPE OPTIMIZATION OF PIEZOELECTRIC SENSORS OR ACTUATORS FOR THE CONTROL OF PLATES
}

\author{
Emmanuel Degryse ${ }^{1}$ and Stéphane Mottelet ${ }^{1}$
}

\begin{abstract}
This paper deals with a new method to control flexible structures by designing noncollocated sensors and actuators satisfying a pseudo-collocation criterion in the low-frequency domain. This technique is applied to a simply supported plate with a point force actuator and a piezoelectric sensor, for which we give some theoretical and numerical results. We also compute low-order controllers which stabilize pseudo-collocated systems and the closed-loop behavior show that this approach is very promising.
\end{abstract}

Mathematics Subject Classification. 49N35, 49Q10, 74P15, 90C47, 93B40, 93C20.

Received August 26, 2002. Revised March 29 and December 25, 2004.

\section{INTRODUCTION}

We focus on systems which can be represented by an abstract controlled wave equation

$$
z_{t t}+\alpha \mathcal{A} z_{t}+\mathcal{A} z=\mathcal{B} v
$$

where $z(x, t), x \in \Omega$, represents the transverse displacement of some flexible structure represented by and open bounded set $\Omega \subset \mathbb{R}^{p}$ ( $p=1$ or 2 ), excited by a scalar control $v(t)$. The system operator $\mathcal{A}$ is a self-adjoint positive operator with compact resolvent on the Hilbert space $H=L^{2}(\Omega)$ and domain $D(\mathcal{A})$. The operator $\mathcal{B}$ is denoted the control operator. The damping coefficient $\alpha$ is positive. We add a scalar observation of the velocity $y=\mathcal{C} z_{t}$ by means of the observation operator $\mathcal{C}$.

In the scope of this introduction, we will consider that $\mathcal{B}$ and $\mathcal{C}$ are bounded operators in $H$, i.e. $\mathcal{B} \in \mathcal{L}(\mathbb{R}, H)$ and $\mathcal{C} \in \mathcal{L}(H, \mathbb{R})$ but in the sequel the equation (1) will be considered to hold in $D(\mathcal{A})^{\prime}$, the dual of $D(\mathcal{A})$, and we will allow $\mathcal{B}$ and $\mathcal{C}$ to be unbounded, i.e. $\mathcal{B} \in \mathcal{L}\left(\mathbb{R}, D(\mathcal{A})^{\prime}\right)$ and $\mathcal{C} \in \mathcal{L}(D(\mathcal{A}), \mathbb{R})$ (piezoelectric or pointwise control and sensing corresponds to unbounded operators).

\subsection{Collocation}

When $\mathcal{C}=\mathcal{B}^{*}$, the sensor and the actuator are said to be collocated. In this case, the feedback $v=-k y$, with $k>0$, provides strong stability of the system (1) (see [5]). This fact can be also understood in the frequency

Keywords and phrases. Collocation, piezoelectric sensors/actuators, positive-real systems, topology optimization.

1 Laboratoire de Mathématiques Appliquées de Compiègne, Département de Génie Informatique, Université de Technologie de Compiègne, BP 20529, 60205 Compiegne Cedex, France; degryse@iutc3.unicaen.fr stephane.mottelet@utc.fr 
domain, because the transfer function associated to the system (1)

$$
G(s)=\sum_{k>0} \frac{\mathcal{C} \varphi_{k} \mathcal{B}^{*} \varphi_{k}}{s^{2}+\alpha \lambda_{k}^{2} s+\lambda_{k}^{2}} s,
$$

where $\left\{\varphi_{k}, \lambda_{k}^{2}\right\}_{k>0}$ are the eigenpairs of $\mathcal{A}$, can be shown to be positive-real,

$$
G(s)>0, \text { for } \operatorname{Re}(s)>0 .
$$

This kind of systems can be stabilized (at least in an input-output sense) by finite dimensional controllers with a strictly positive-real transfer function $K(s)$ through negative feedback (see $[5,28]$ ). It is well known that the constant velocity feedback $v=-k y$, with $k>0$ can be highly non robust with respect to delays in the feedback path for some boundary control systems (see e.g. [8], Sect. 5.8, and [7], for a general discussion about this problem). Moreover, the feedback $v=-k y$ is quite unrealistic in the applications since it does not take into account the eventual presence of noise in the observation. This is why dynamic controllers with a strictly proper transfer function $\left(\lim _{f \rightarrow \infty}|K(j f)|=0\right)$ are preferred (see [26] for an example).

One can also use LQG or $\mathrm{H}_{\infty}$ controllers (see [14,21] for a general discussion, and [2] for applications to the control of structures with piezoelectric devices). These controllers offer much freedom in the design but do not have a strictly-positive real transfer function in general. In any case, the property (3) is still relevant for these kinds of controllers, because it guarantees that the transfer function $G(s)$ has no zeros in the right-half plane. For example, when solving a mixed sensitivity problem, property (3) ensures that the sensitivity function can be made arbitrary small in the bandwidth of the system (see [11,13]). It has also been shown in $[18,30]$ that the input-output behavior of non-collocated systems can be ill-conditioned with respect to uncertainty in the sensor and actuator positions.

Unfortunately collocation is very rare in practice, because the relationship $\mathcal{C}=\mathcal{B}^{*}$ implies that the sensor and the actuator are located at the same position on the structure (see Prop. 1.1 below). In most cases, either it is not possible to sense and actuate at the same location for technological reasons, or one of the two operators does not correspond to any implementable device. In the following section we show that the collocation property can be relaxed in practical issues.

\subsection{Pseudo-collocation criterion}

The following analysis can be done: when $\mathcal{C}=\mathcal{B}^{*}$, the following inequality is verified

$$
\mathcal{C} \varphi_{k} \mathcal{B}^{*} \varphi_{k}>0, \quad k>0 .
$$

It is now well understood that in most cases, even if $\mathcal{C} \neq \mathcal{B}^{*}$, the property (4) is itself sufficient to prove strong stability, by the application of the La Salle's invariance principle (see [15]), but a careful analysis is sometimes necessary when $\mathcal{B}$ and/or $\mathcal{C}$ are unbounded (see e.g. $[22,27]$ ). In general, it can be easily shown that (4) implies that the transfer function (2) is positive-real, but the necessity of placing the sensor and the actuator at the same location still remains at least when $\mathcal{B}$ and $\mathcal{C}$ are bounded, as shown in the following result:

Proposition 1.1. Suppose that $\mathcal{A}$ is a self-adjoint positive operator with compact resolvent on a Hilbert space $H$. Let $\mathcal{B} \in \mathcal{L}(\mathbb{R}, H)$ and $\mathcal{C} \in \mathcal{L}(H, \mathbb{R})$ be two bounded operators in $H$. Let $\left\{\varphi_{k}\right\}_{k>0}$ be the (orthogonal) eigenfunctions of $\mathcal{A}$. Then the property

$$
\mathcal{C} \varphi_{k} \mathcal{B}^{*} \varphi_{k}>0, \quad k>0,
$$

implies that $\mathcal{B}$ and $\mathcal{C}$ cannot have disjoint supports.

Proof. Since $\mathcal{B} \in \mathcal{L}(\mathbb{R}, H)$ and $\mathcal{C} \in \mathcal{L}(H, \mathbb{R})$ are bounded there exists $b, c \in H$ such that $\mathcal{C} z=\langle c, z\rangle$ and $\mathcal{B}^{*} z=\langle b, z\rangle$. If $\mathcal{B}$ and $\mathcal{C}$ had disjoint supports we would have $\langle b, c\rangle=0$, but

$$
\langle b, c\rangle=\sum_{k>0}\left\langle c, \varphi_{k}\right\rangle\left\langle b, \varphi_{k}\right\rangle=\sum_{k>0} \mathcal{C} \varphi_{k} \mathcal{B}^{*} \varphi_{k}>0 .
$$


The idea to be developed is that the relationship (4) is too strong for practical issues. The control objectives usually concern the low frequency behavior of the system because the model of flexible structures is rather inexact in the high frequency range. This motivates the idea that (4) can be weakened to

$$
\mathcal{C} \varphi_{k} \mathcal{B}^{*} \varphi_{k}>0, \quad K \geq k>0
$$

The interest of this approach is that the relationship (6) does not prevent $\mathcal{B}$ and $\mathcal{C}$ to have disjoint supports since $K$ is finite. The property (6) makes senses also in the unbounded case, at least when there exists integers $n$ and $m$ such that $\mathcal{C} \in \mathcal{L}\left(D\left(\mathcal{A}^{n}\right), \mathbb{R}\right)$ and $\mathcal{B} \in \mathcal{L}\left(\mathbb{R}, D\left(\mathcal{A}^{m}\right)^{\prime}\right)$ (this is the case if $\mathcal{B}$ and $\mathcal{C}$ are made of Dirac distributions or partial derivatives of Dirac distributions concentrated in interior points or curves in $\Omega$ ). Hence, the idea that we will develop is that the property (6) can be used to propose a criterion to optimize the sensor (and/or actuator) shape and location with the constraint that $\mathcal{B}$ and $\mathcal{C}$ have disjoint supports.

A similar criterion has been recently applied for mechanical systems by Hara, Iwasaki and Shimizu [29]: the FFPR property (Finite Frequency Positive Real) is used for designing the profile of piezoelectric films on a smart arm structure. However, the design methods are based on the linear matrix inequalities whereas what follows is mainly based on classical optimization tools (linear programming) and allows to deal with more complex shapes.

\subsection{Remarks on closely related problems}

When a constant feedback $u=-2 \varepsilon y$ is used, (1) is equivalent to the closed-loop system

$$
z_{t t}+(\alpha \mathcal{A}+2 \varepsilon \mathcal{D}) z_{t}+\mathcal{A} z=0, x \in \Omega
$$

with a damping operator $\mathcal{D}=\mathcal{B C}$. If we consider $\lambda_{k}(\varepsilon)$ the $k$ th eigenvalue of the operator

$$
A=\left(\begin{array}{cc}
0 & I \\
-\mathcal{A} & -\alpha \mathcal{A}-2 \varepsilon \mathcal{D}
\end{array}\right),
$$

then the quantity $-\left\langle\mathcal{D} \varphi_{k}, \varphi_{k}\right\rangle=-\mathcal{C} \varphi_{k} \mathcal{B}^{*} \varphi_{k}$ is equal to $\frac{\partial \lambda_{k}}{\partial \varepsilon}(0)$ (see [20], pp. 421, 422), and measures, in some sense and for small values of $\varepsilon$, the efficiency of the feedback for the $k$ th eigenmode of the system (7). This particular case is related to the one studied in [12], where the authors consider a one-dimensional wave equation in the hyperbolic case $\left(\alpha=0\right.$ and $\left.\mathcal{A}=-\frac{\partial^{2}}{\partial x^{2}}\right)$ and a damping operator $\mathcal{D}$ given by $(\mathcal{D} z)(x)=a(x) z(x)$, where $a(x)$ is not necessary non-negative. In this paper, the authors show that the quantities $\left\langle\mathcal{D} \varphi_{k}, \varphi_{k}\right\rangle$ have to be positive for all $k>0$ and bounded away from zero to ensure exponential stability of (7) for small values of $\varepsilon$.

Trying to maximize the lower bound of these quantities with respect to design parameters of $\mathcal{D}$ seems rather natural. In [16] the authors consider the case $(\mathcal{D} z)(x)=\chi_{\omega}(x) z(x)$, where $\chi_{\omega}$ is the characteristic function of some open set $\omega \subset \Omega$, and study the maximization of this lower bound with respect to $\omega$ (for the one-dimensional wave equation). In the next section we will also propose a similar criterion, which will be limited to a finite number of eigenmodes, because of the constraint that $\mathcal{B}$ and $\mathcal{C}$ have disjoint supports.

Considering a finite number of eigenmodes could seem questionable (see e.g. [17]), but we insist on the fact that we consider the parabolic case $(\alpha>0)$ and that the constant feedback $u=-2 \varepsilon z_{t}$ is never used in control engineering, as explained in Section 1.1. In Section 3 we will show how finite dimensional controllers can be used to solve the stability problem. Thus, the spillover problem is not an issue here. But the performances of the controller on the other eigenmodes could be taken in account in the optimization. We will give some elements in that direction in Section 6. 


\section{Sensor Shape optimization for a plate EqUation}

\subsection{The model}

The system is a classical simply supported plate equation with Kelvin-Voigt damping

$$
\begin{aligned}
z_{t t}+\alpha \Delta^{2} z_{t}+\Delta^{2} z & =\mathcal{B} v, x \in \Omega, t>0 \\
z=\Delta z & =0, x \in \partial \Omega
\end{aligned}
$$

where $\Omega \subset \mathbb{R}^{2}$ is some open set.

The observation $y$ is obtained through a piezoelectric sensor (with normalized piezoelectric constants) by $y=\mathcal{C} z_{t}$, where

$$
\mathcal{C} z=\int_{\omega} \Delta z \mathrm{~d} x=\int_{\partial \omega} \partial_{n} z \mathrm{~d} \gamma
$$

where the domain $\omega$ represents the area covered by the piezoelectric material on the plate. Because of the reciprocity of piezoelectric effect, the case of a fixed observation operator and a couple of identical facing piezoelectric actuators driven out of phase (thus generating pure bending moments) is equivalent (see [10] for a discussion on the modeling of piezoelectric devices).

The problem that we will study is closely related to the problems discussed in [19,24]. In these papers the authors discuss the stabilization and the controllability of systems of the type (8) by means of dampers localized in the contour of a domain. This kind of damping corresponds to a sensor and an actuator both of piezoelectric type, located at the same position on the structure, in the typical case $\mathcal{B}^{*}=\mathcal{C}$, that we do not consider here, as we have explained in Sections 1.1 and 1.2.

The problem of finding $\omega$ such that (6) is satisfied can be formulated as follows: find $\omega_{\text {opt }}$ such that

$$
j\left(\omega_{\text {opt }}\right)=\max _{\omega \subset \Omega} j(\omega), \quad \text { with } j(\omega)=\min _{k=1 \ldots K} w_{k} \mathcal{C} \varphi_{k} \mathcal{B}^{*} \varphi_{k}
$$

where the functions $\left\{\varphi_{k}\right\}_{k>0}$ are the eigenfunctions of $\Delta^{2}$ with boundary conditions (9) and $w_{k}>0$. It is well known that the particular form of (9) implies that the $\left\{\varphi_{k}\right\}_{k>0}$ are also the eigenfunctions of $-\Delta$ with Dirichlet boundary condition. We will assume that $\mathcal{B}$ is such that $\mathcal{B}^{*} \varphi_{k}=b_{k} \neq 0$, for $k=1 \ldots K$ (the first $K$ eigenmodes are controllable).

With the weight $w_{k}=\lambda_{k}^{-2}$, where $\lambda_{k}$ is the $k$ th eigenvalue of $-\Delta$ with Dirichlet boundary condition, the quantity $w_{k} \mathcal{C} \varphi_{k} \mathcal{B}^{*} \varphi_{k}$ represents the peak value on the imaginary axis (up to the multiplicative constant $\alpha^{-1}$ ) of the modulus of the $k$ th term in the sum (2). Of course, these weights can be chosen differently, allowing to amplify or attenuate the contribution of a particular mode of the plate in the frequential response of the system.

When simply connected domains are considered the shape optimization problem can be formulated as: find $\omega_{\text {opt }}$ such that

$$
\tilde{\jmath}\left(\omega_{\text {opt }}\right)=\max _{\partial \omega \in \mathcal{G}} \tilde{\jmath}(\omega), \quad \text { with } \tilde{\jmath}(\omega)=\min _{k=1 \ldots K} b_{k} \int_{\partial \omega} \partial_{n} \varphi_{k} \mathrm{~d} \gamma
$$

where $\mathcal{G}$ is a set of admissible curves. A numerical study of this non-linear problem can be done when $\mathcal{G}$ is a finite dimensional space (e.g. a set of polygonal curves). But reliable results are difficult to obtain because the problem is ill-conditioned. Moreover the choice of the class of simply connected domains is arbitrary since the topology of $\omega_{\text {opt }}$ is unknown a priori.

In the following we will prove an existence and uniqueness result of the solution of (10) by considering a relaxed version of this problem. 


\subsection{Main results}

In this section we consider, without loss of generality, that $w_{k}=1, \forall k$. We also consider the set $\mathcal{U}=\{f \in$ $H / 0 \leq f \leq 1$ in $\Omega\}$, and the following linear problem: find $f_{\text {opt }}$ such that

$$
J\left(f_{\text {opt }}\right)=\min _{f \in \mathcal{U}} J(f), \quad \text { with } J(f)=-\min _{k=1 \ldots K} b_{k} \int_{\Omega} f \Delta \varphi_{k} \mathrm{~d} x,
$$

which is a relaxed version of (10) since $f$ is not constrained to be the characteristic function of some open set $\omega \in \Omega$. The intermediate values of $f$ could be penalized by adding to the above functional a term of the type

$$
\psi(f)=-\frac{1}{\epsilon} \int_{\Omega} f(f-1) \mathrm{d} x
$$

therefore forcing $f$ to approach the characteristic function of some subdomain $\omega$ of $\Omega$ as $\epsilon \rightarrow 0^{+}$, but we will see in the following that we don't need this penalizing term.

The idea of replacing the initial unknown, which is an open set, by a function $f$ tending to a characteristic function, is a classical technique that is used in topological optimization (see e.g. [1]). We give an existence result:

Theorem 2.1. The problem (12) admits an optimal solution.

Proof. The set $\mathcal{U}=\{f \in H / 0 \leq f \leq 1$ in $\Omega\}$ is bounded and closed in the Hilbert space $H$, therefore weakly compact. Moreover the functional $J$ defined on $H$ by

$$
J(f)=-\min _{k=1 \ldots K} b_{k} \int_{\Omega} f \Delta \varphi_{k} \mathrm{~d} x
$$

is weakly continuous on $H$. This is enough to claim that the problem (12) admits a solution in $\mathcal{U}$.

Note that in the above proof $J$ and $\mathcal{U}$ are convex. The following result shows that the solution is unique and that the penalizing term is useless.

Theorem 2.2. Consider the problem (12). Then the optimal function $f$ is unique and has necessarily the property

$$
f(f-1)=0, \text { a.e. in } \Omega .
$$

Proof. We put (12) under a differentiable form by introducing a new scalar variable $\eta$,

$$
\min _{f, \eta}-\eta
$$

under the constraints

$$
\begin{aligned}
\eta-b_{k} \int_{\Omega} f \Delta \varphi_{k} \mathrm{~d} x & \leq 0, \quad k=1 \ldots K \\
-f(x) & \leq 0 \\
f(x) & \leq 1 .
\end{aligned}
$$

We introduce the spaces $X=\mathbb{R} \times L^{2}(\Omega)$ and $Z=\mathbb{R}^{K} \times L^{2}(\Omega) \times L^{2}(\Omega)$ and we define an inner product on $Z$ as

$$
\langle\chi, \zeta\rangle_{Z}=\sum_{i=1}^{K} \chi_{i} \zeta_{i}+\left\langle\chi_{K+1}, \zeta_{K+1}\right\rangle+\left\langle\chi_{K+2}, \zeta_{K+2}\right\rangle
$$


The space $Z$ together with this inner product is an Hilbert space. We also define the following mapping $T$ from $X$ into $Z$

$$
\begin{aligned}
T: X & \longrightarrow Z \\
(\eta, f) & \longmapsto\left(\eta-b_{1}\left\langle f, \Delta \varphi_{1}\right\rangle, \ldots, \eta-b_{K}\left\langle f, \Delta \varphi_{K}\right\rangle,-f, f-1\right) .
\end{aligned}
$$

If a pair $t=(\eta, f)$ is a regular solution of our problem, then it satisfies the Kuhn-Tucker conditions (see [23]): there is a $\zeta \in Z$ such that the Lagrangian

$$
L(t, \zeta)=-\eta+\langle T(t), \zeta\rangle_{Z}
$$

is stationary at $t$. By setting $\zeta=\left(p_{1}, \ldots, p_{K}, q_{1}, q_{2}\right)$, we obtain

$$
L(\eta, f, \zeta)=-\eta+\sum_{k=1}^{K} p_{k}\left(\eta-b_{k}\left\langle f, \Delta \varphi_{k}\right\rangle\right)+\left\langle f-1, q_{1}\right\rangle-\left\langle f, q_{2}\right\rangle
$$

We have

$$
\begin{aligned}
\frac{\partial L}{\partial \eta} & =-1+\sum_{k=1}^{K} p_{k} \\
\frac{\partial L}{\partial f} \cdot g & =-\sum_{k=1}^{K} b_{k} p_{k}\left\langle g, \Delta \varphi_{k}\right\rangle+\left\langle g, q_{1}\right\rangle-\left\langle g, q_{2}\right\rangle \\
& =\left\langle-\sum_{k=1}^{K} b_{k} p_{k} \Delta \varphi_{k}+q_{1}-q_{2}, g\right\rangle
\end{aligned}
$$

The optimality system for the problem (15)-(17) is given by the Kuhn-Tucker conditions:

$$
\begin{aligned}
\sum_{k=1}^{K} p_{k} & =1, \\
-\sum_{k=1}^{K} b_{k} p_{k} \Delta \varphi_{k}+q_{1}-q_{2} & =0, \\
q_{1}(f-1)=q_{2} f & =0
\end{aligned}
$$

with $p \geq 0$ and $q_{1} \geq 0, q_{2} \geq 0$, in $\Omega$. Suppose now that $f$, a solution of the above system, is such that

$$
\exists \mathcal{O} \subset \Omega, f(f-1) \neq 0, \text { in } \mathcal{O}
$$

where $\operatorname{mes}(\mathcal{O}) \neq 0$. In this case we have $f \neq 0$ and $(f-1) \neq 0$, in $\mathcal{O}$ so that $q_{1}=q_{2}=0$ in $\mathcal{O}$ and as a consequence

$$
\sum_{k=1}^{K} b_{k} p_{k} \Delta \varphi_{k}=-\sum_{k=1}^{K} b_{k} p_{k} \lambda_{k} \varphi_{k}=0, \text { in } \mathcal{O}
$$

since $-\Delta \varphi_{k}=\lambda_{k} \varphi_{k}$. Since the functions $\left\{\varphi_{k}\right\}_{k=1 \ldots K}$ are analytic the above finite sum is also analytic, thus vanishes in the whole domain $\Omega$. This implies that $p=0$ because $b_{k} \lambda_{k} \neq 0$. This contradicts with $\sum_{k=1}^{K} p_{k}=1$. We have finally the result:

$$
f(f-1)=0, \text { in } \Omega .
$$

To show that $f$ is unique, we first note that in the problem $(12) J$ is convex (but not strictly convex) and $\mathcal{U}$ is also convex. Suppose now that there exists $f$ and $g$ two distinct solutions of (12), thus necessarily verifying 
$f(f-1)=0$ and $g(g-1)=0$. Since $\mathcal{U}$ is convex every function $f_{\lambda}$ of the form $f_{\lambda}=\lambda f+(1-\lambda) g$, for $\left.\lambda \in\right] 0,1[$ must also be a solution. Then it is easy to show that $f \neq g$ implies that $f_{\lambda}=\lambda$ or $f_{\lambda}=1-\lambda$, in some open set of $\Omega$, thus contradicting (14).

This result shows that the optimal $f$ is necessarily the characteristic function of some open set $\omega \in \Omega$. Since the problem formulation places no assumptions on the number of holes cut within the domain, it can be seen as a topology optimization problem. We will see in the numerical results that the optimal $\omega$ such that $f(x)=\mathbf{1}_{\omega}(x)$ may be not connected in some cases.

Remark 2.3. It can be shown that the property (14) still holds if we force $f$ to vanish in any subdomain of $\Omega$ : the proof is quite the same as previously and can be found in [9]. This is a crucial result since we want to design non-collocated sensors and actuators.

Remark 2.4. For technological reasons, the size of the sensor may be limited, thus we may need to modify our problem by adding the constraint

$$
\int_{\Omega} f \mathrm{~d} x \leq \sigma, \quad(\sigma>0)
$$

which restricts the area covered by the sensor. Under this additional constraint, the property (14) remains valid as shown in [9].

Remark 2.5. From an engineering point of view, $K$ will be eventually large, but the needed control bandwidth will always be finite, because structural models are very inexact in the high frequency range, because of actuator bandwidth limitations, etc. However, a possible question to address is the behavior of $\omega$ as $K \rightarrow \infty$

If the disjoint support hypothesis is kept, we know that it is impossible to have

$$
\mathcal{C} \varphi_{k} \mathcal{B}^{*} \varphi_{k}>0, \forall k>0
$$

thus we will have necessarily $\lim _{K \rightarrow \infty} \eta=0$ and $\lim _{K \rightarrow \infty} \operatorname{mes}(\omega)=0$. We have a confirmation of this property in the numerical results.

If the disjoint support constraint is relaxed (we recall that this constraint is motivated by practical considerations), we don't have a complete answer, but we can anyway give some elements. We can mention a closely related shape optimization problem (in one dimension, see [16]) where $\mathcal{C}=\mathcal{B}^{*}$ and a similar criterion is optimized with respect to a one-dimensional domain $\omega$. In this paper the authors show that if $\omega$ is searched as the union of a finite number of intervals, then the number of intervals must be fixed a priori, otherwise an optimum does not exist.

\section{Stabilization By Finite Dimensional CONTROLleRs}

We will show here why the criterion we have used in the optimization leads to systems that have interesting input-output properties allowing to obtain finite dimensional (i.e. governed by an ordinary differential equation) controllers, allowing to stabilize the system without spillover.

In this section we reformulate the plate equations (8)-(9) under a first order (in time) form: the new state $x=\left(z, z_{t}\right)$ satisfies

$$
\left\{\begin{aligned}
x_{t} & =A x+B v \\
y & =C x
\end{aligned}\right.
$$

with $A x=\left(x_{2},-\alpha \Delta^{2} x_{2}-\Delta^{2} x_{1}\right), B v=(0, \mathcal{B} v)$ and $C x=\mathcal{C} x_{2}$.

An adequate framework for control synthesis is the Pritchard-Salamon class of state-space systems, together with the Callier-Desoer algebra of transfer functions $\hat{\mathcal{B}}(0)$ (see [7] for an excellent survey). The system (8)-(9) can be shown to belong to the Prichard-Salamon class, by using a classical semigroup approach and some regularity results (see [10] and particularly [31] for sharp results). Moreover the transfer function

$$
G(s)=C(s I-A)^{-1} B .
$$


exists and belongs to $\hat{\mathcal{B}}(0)$ because the operator $A$ generates an exponentially stable semigroup (see [6] Th. 4.1), due to the Kelvin-Voigt damping. This allows to give a sense to the expansion

$$
G(s)=\sum_{k>0} \frac{\mathcal{C} \varphi_{k} \mathcal{B}^{*} \varphi_{k}}{s^{2}+\alpha \lambda_{k}^{2} s+\lambda_{k}^{2}} s .
$$

We now consider a triplet $(A, B, C)$ where $C$ represents a piezoelectric sensor covering the domain $\omega$ obtained as the solution of the problem (12) for a given $K>0$. We can write

$$
G(s)=G_{K}(s)+\Delta_{K}(s)
$$

where

$$
G_{K}(s)=\sum_{k=1}^{K} \frac{\mathcal{C} \varphi_{k} \mathcal{B}^{*} \varphi_{k}}{s^{2}+\alpha \lambda_{k}^{2} s+\lambda_{k}^{2}} s .
$$

The transfer function $G_{K}(s)$, by construction, is positive real, and $\Delta_{K}(s)$ can be considered here as an additive perturbation. Let us consider a controller, represented by its transfer function $P(s)$, exponentially stabilizing $G_{K}(s)$. The controller $P(s)$ can be a constant $p>0$, or of the form

$$
P(s)=\frac{p}{s+s_{0}},
$$

with $p>0$ and $s_{0}>0$. These two examples are positive real transfer matrices, and they exponentially stabilize $G_{K}(s)$, whatever the value of $K$. The controller $P(s)$ can also stabilize $G(s)$, as shown in the following theorem (see [4]):

Theorem 3.1. Let $G$ and $\Delta$ be two transfer functions in $\hat{\mathcal{B}}(0)$. Suppose that

- $G$ is strictly proper $\left(\lim _{f \rightarrow \infty}|G(j f)|=0\right)$ and has no poles on the imaginary axis;

- $\Delta$ is strictly proper and $\|\Delta\|_{\infty} \leq \varepsilon$

- $G$ and $G+\Delta$ have the same number of unstable poles;

A controller $P$ stabilizing $G$ will also stabilize $G+\Delta$ if

$$
\left\|P(I+G P)^{-1}\right\|_{\infty} \leq \frac{1}{\varepsilon} .
$$

In the above theorem, we have used the notation $\|G\|_{\infty}=\sup _{f}|G(j f)|$. This theorem allows to claim that it is always possible to stabilize the plate. In fact, for any (finite dimensional) controller $P(s)$ stabilizing $G_{K}(s)$, there will always exist a controller of the form $g P(s)$ stabilizing $G(s)$, because the inequality

$$
\left\|\Delta_{K}\right\|_{\infty} \leq\left\|(g P)^{-1}+G_{K}\right\|_{\infty},
$$

can always be verified if $g>0$ is sufficiently small. It is straightforward to see in this inequality the tradeoff between the norm of the perturbation $\Delta_{K}$ and the gain of the controller. The smaller the norm of the perturbation, the bigger the gain can be taken without loosing stability. To this extent, the constraints in the optimization problem (12) could be modified to control the norm of $\Delta_{K}$ (see Sect. 6).

\section{NUMERICAL RESULTS}

\subsection{Approximation of the optimal sensor shape}

In this example, the operator $\mathcal{B}$ is chosen to represent a point force actuator, i.e. $\mathcal{B}^{*} z=z(a)$ where $a \in \Omega$ is the point where the force is applied on the structure. We add the constraint that $f$ vanishes on a small disc 

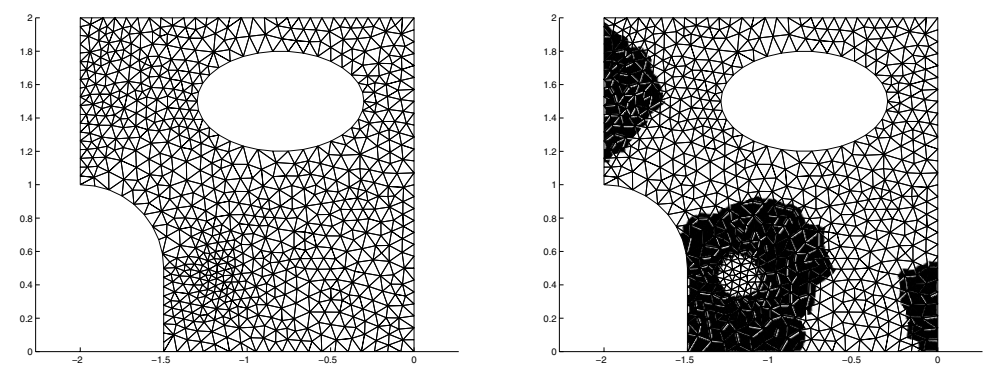

Figure 1. Structure mesh and optimal sensor for $n=767, K=8$. Actuator is at $(-1.23191,0.421925)$.

with center $a$ (see Rem. 2.3). This constraint takes the form

$$
\int_{D(a, R)} f \mathrm{~d} x=0
$$

The numerical approximation of (12) involves the approximation of the eigenfunctions $\varphi_{k}$ and eigenvalues $\lambda_{k}^{2}$ of $\mathcal{A}=\Delta^{2}$ with $\Delta z=z=0$ boundary conditions on a triangular mesh. We also need to approximate the functions $\Delta \varphi_{k}$. Since $\mathcal{A}^{\frac{1}{2}}$ is the operator $-\Delta$ with Dirichlet boundary conditions, we only need to use a subspace $V_{h}$ of $C_{0}$ piecewise linear elements based on a triangulation of $\Omega$.

Each function $f$ in $V_{h}$ is completely determined by its nodal values $f_{k}$, and the eigenpairs of $-\Delta$ with Dirichlet boundary condition are approximated by solving the eigenvalue problem $S y^{k}=\lambda_{k} M y^{k}$, where $S$ is the stiffness matrix and $M$ is the mass matrix. The original problem (12) is approximated by the linear program

$$
\begin{gathered}
\min _{f, \eta}-\eta, \\
\eta-b_{k} \lambda_{k}\left(y^{k}\right)^{\top} M f \leq 0, \quad k=1 \ldots K \\
0 \leq f_{p} \leq 1, \quad p=1 \ldots n,
\end{gathered}
$$

where $n$ is the number of nodes in the discretization of $\Omega$. We use Freefem to construct the mesh and Matlab for the rest of the computations. The linear program has been solved using a primal-dual interior point algorithm (see [32]). The Figure 1, where the sensor is painted in black, shows the results for a coarse mesh $(n=767)$ and $K=8$. The optimal value of $\eta$ is 3.60. The Figure 2 shows the approximation of the transfer function: clearly the system behaves like a positive-real system in a frequency band corresponding to the first $K$ natural frequencies (the phase is in the interval $[-90,90])$.

Remark 4.1. A question that can be addressed is about the convergence of the approximation of the sensor, as $n$ increases, to the solution of the infinite-dimensional problem. In [9] the sensors computed for greater values of $n$ let us suppose that the solution of the discretized problem converges: we can notice the regularization of the shape of the domain $\omega$. However, theoretical results about this problem have not been shown yet. The linearity of the optimization problem allows us to place in the framework of Theorem 10 in [25], p. 207, but in the latter, the first assumption is too strong for our case (the second is readily satisfied), thus it cannot be used in this form.

Anyway, we think that the reformulation of (10) under the relaxed form (12) is not helpful to show the convergence of the finite element approximation of the sensor. In fact, a recent result (see [3]) shows the convergence of the finite element approximation of a shape optimization problem involving the solution of an elliptic problem. Although this result deals with a particular problem, the approach which is used seems to be general enough to be applied to many shape optimization problems. We will study the application of this result to our problem in a forthcoming paper. 

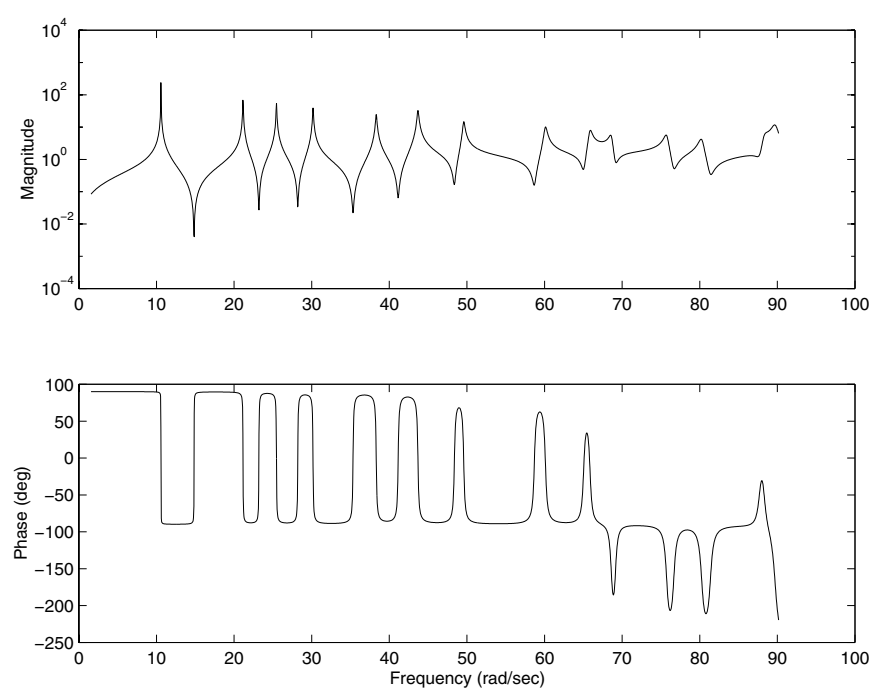

Figure 2. Bode diagram of the system transfer function with the optimal sensor.

TABLE 1. Values of $\eta$ corresponding to distorted sensors (10000 deformations).

\begin{tabular}{|c||c|c|c|}
\hline & $n=767$ & $n=1587$ & $n=4873$ \\
\hline \hline Optimal value of $\eta$ & 3.60 & 3.81 & 3.94 \\
\hline \hline Minimal value of $\eta$ & 0.98 & 2.27 & 3.11 \\
\hline Average value of $\eta$ & 2.76 & 3.29 & 3.69 \\
\hline Maximal value of $\eta$ & 3.69 & 4.01 & 4.03 \\
\hline
\end{tabular}

\subsection{Behavior of pseudo-collocated systems with respect to shape uncertainties}

We have designed an optimal sensor in order to make our system positive-real in the low-frequency domain. however, in practice, it will be difficult to create exactly the optimal sensor. So we need to know if an approximate shape of this sensor is sufficient to have a pseudo-collocated system. We have modified the optimal shape and recalculated the corresponding $\eta$. The following table shows the results obtained for three types of mesh. The first line is the optimal value of $\eta$ computed in Section 4.1. the others are respectively the minimal, average and maximal values of $\eta$ obtained by distorting the sensor, 10000 deformations have been computed.

Table 1 shows that, even on a coarse mesh, the modified system remains pseudo-collocated. In fact modifying slightly the shape of the sensor is typically a high-frequency disturbance. In practice, this allows us to approximate the optimal sensor with a simpler one, e.g. polygonal or smooth, without losing the property of pseudo-collocation of the system.

Remark 4.2. When deformations are computed, it happens that the value of $\eta$ which is obtained exceeds the optimal value. It comes from the fact that the constraint for the shape to vanish on a disc around the actuator is not necessarily respected.

\subsection{Influence of the number of controlled modes}

Here, we extend the bandwidth on which we insure the positivity of the system. First, it is interesting to see that increasing $K$ does not increase significantly the number of constraints (and the calculation time) if 


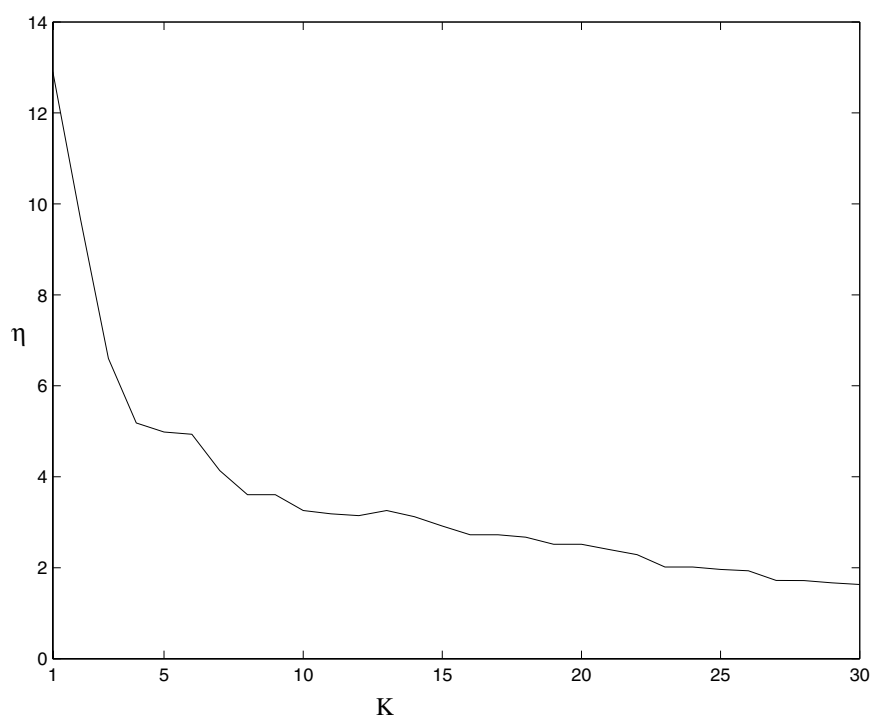

Figure 3. Influence of $K$ on the value of $\eta$.

we compare to the $2 n$ bound constraints. Figure 3 shows the decreasing of $\eta$ when $K$ increases. Let us notice that for high values of $K$, the system might be more sensitive to shape uncertainties of the sensor as seen in Section 4.2 .

\subsection{Influence of the area constraint}

As seen previously (Rem. 2.4), the main theoretical results remain valid if we add an area constraint limiting the surface covered by the sensor. The discretized form of (24) is

$$
d M \tilde{f} \leq \alpha \sigma_{0},
$$

where $d=(1, \ldots, 1), 0<\alpha \leq 1$ and $\sigma_{0}$ is the surface of the plate.

The influence of $\alpha$ on the solution of the problem is studied. Figure 4 shows that the value of $\eta$ increases with $\alpha$ until the constraint (31) becomes inactive. Additionally, it could be expected that diminishing the maximal area of the sensor would lead to a reduction of the number of connected components of $\omega$. This would be interesting for technological reasons, but this is not the case as shown in Figure 5. The area constraint acts on each component simultaneously.

\section{DESIGN OF FINITE-DIMENSIONAL CONTROLLERS FOR PSEUDO-COLLOCATED SYSTEMS}

\subsection{LQG control}

To validate the sensor shape, we use a LQG controller built on a projection of the discretized system on the first $N=32$ eigenmodes. Figure 6 represents the eigenvalues of the closed-loop matrix, and we see that the eigenvalues corresponding to the first $K$ frequencies are well shifted to the left. Another way to evaluate the performance of the controller is to compute the mean value of the transfer function from a uniformly distributed random exogenous pointwise force to the natural energy of the system $E\left(z, z_{t}\right)=\|\Delta z\|_{H}^{2}+\left\|z_{t}\right\|_{H}^{2}$, which is given in Figure 7 . We see that none of the first $K$ modes is missed by the controller. 


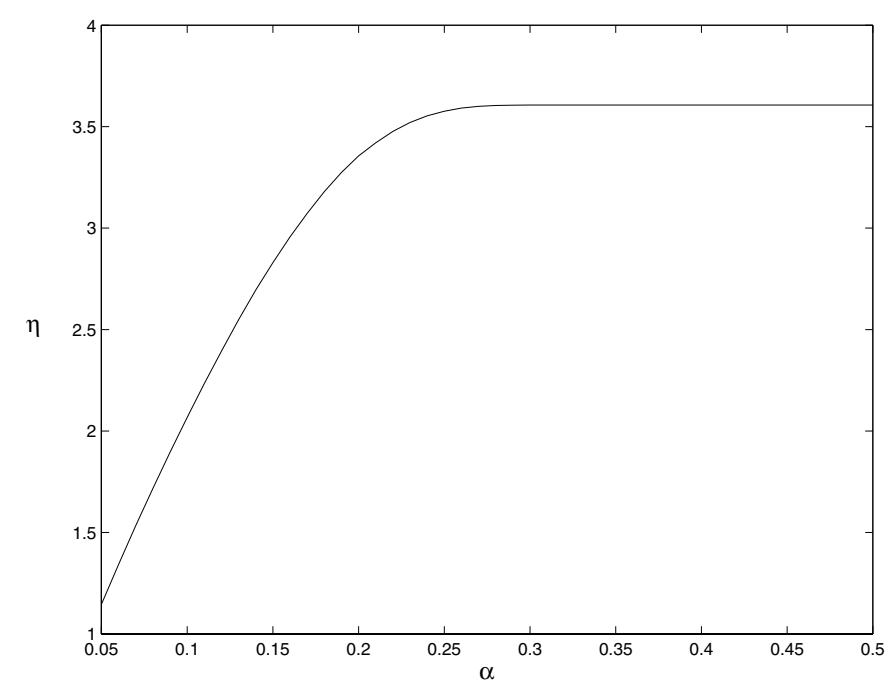

FIGURE 4. Influence of the area constraint on the solution of the problem.
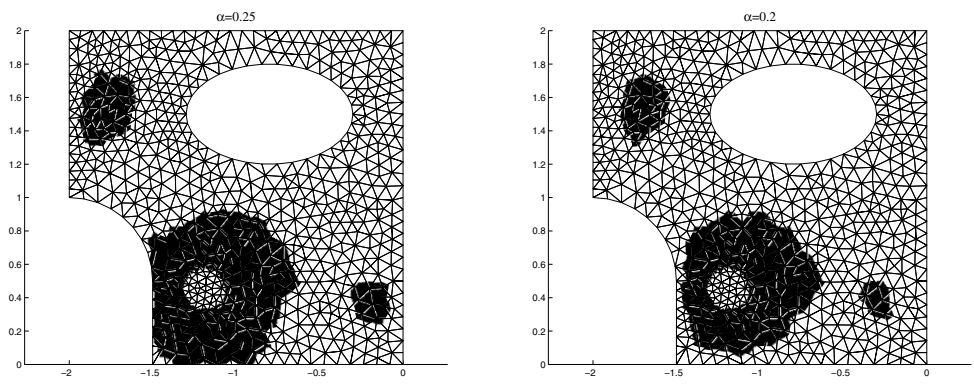

FiguRE 5. Optimal sensor obtained for $\alpha=0.25$ and $\alpha=0.2$.

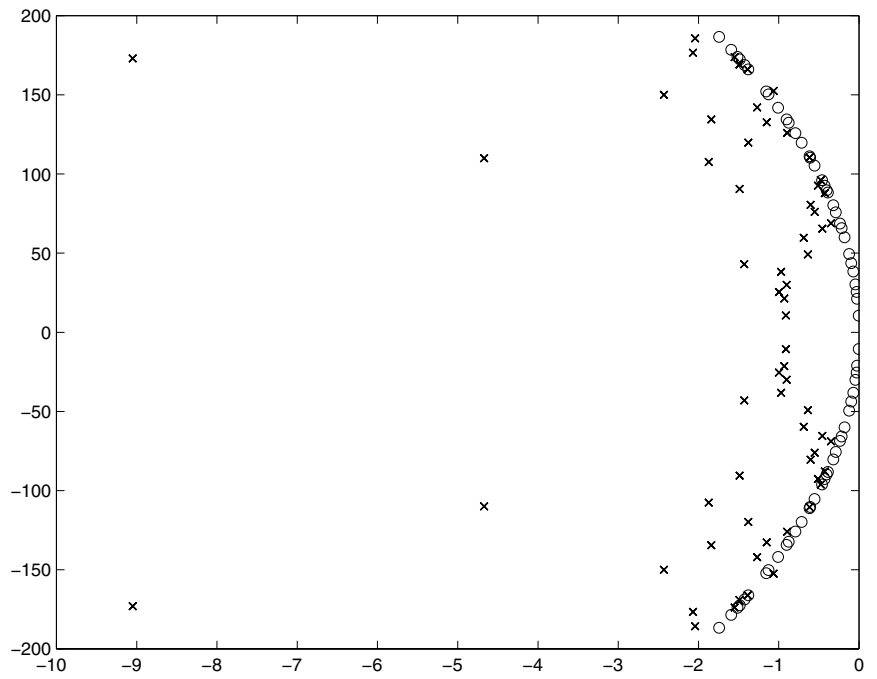

Figure 6. Poles of the open-loop (o) and closed-loop system (x). 


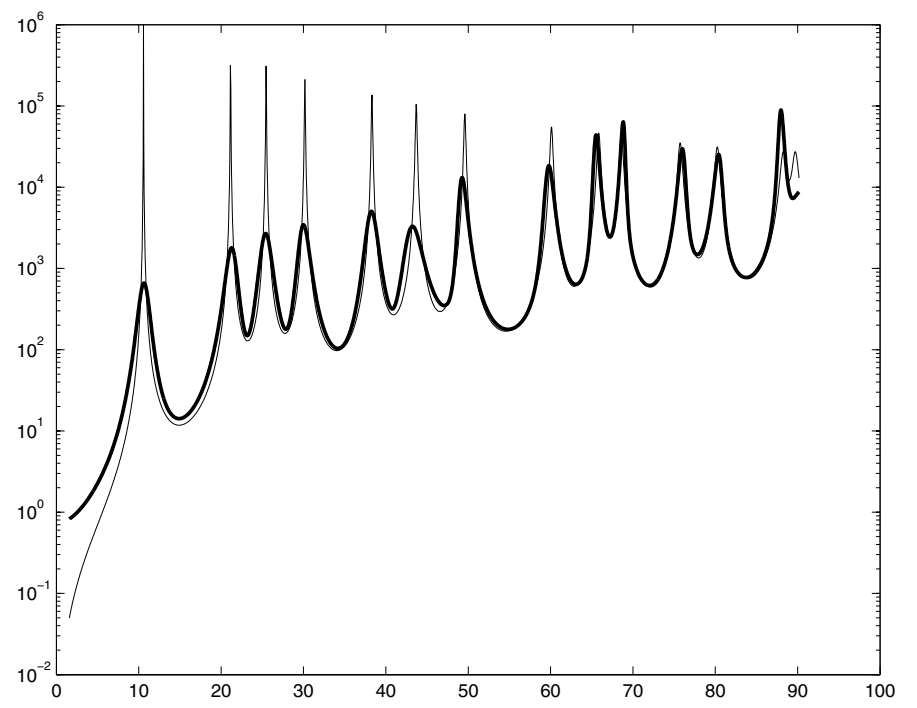

FIGURE 7. Magnitude of the mean energy with respect to the frequency of exogenous input, with (thick line) and without feedback (thin line).

\subsection{Stabilization by a low-pass controller}

The transfer function $G(s)$ can be written

$$
G(s)=G_{K}(s)+\Delta_{K}(s)
$$

where

$$
G_{K}(s)=\sum_{k=1}^{K} \frac{\mathcal{C} \varphi_{k} \mathcal{B}^{*} \varphi_{k}}{s^{2}+\alpha \lambda_{k}^{2} s+\lambda_{k}^{2}} s,
$$

can be shown to be positive-real. We now try to use this particular form for the design of low-order controllers. It is well known that positive-real systems can be stabilized by positive-real systems through negative feedback. For example, $G_{K}(s)$ can be stabilized by a static velocity feedback $v=-g_{0} y\left(g_{0}>0\right)$. However, this type of feedback is known to be non-robust with respect to delays in the feedback path (see [8]) and so we prefer to consider low-pass controllers of the form

$$
L(s)=g_{0} \frac{\omega_{c}}{s+\omega_{c}}
$$

which are strictly positive-real. The cut-off frequency $w_{c}$ must not be chosen too small so that the controller can act on all the pseudo-collocated modes and the static gain $g_{0}$ must be adjusted so that $L(s)$ stabilizes $G(s)$, i.e. is robust with respect to the additive perturbation $\Delta_{K}(s)$.

In our application, $G(s)$ is the system considered in 5.1. The values $g_{0}=0.3$ and $\omega_{c}=25$ seem to be a good compromise here between performance and robustness as shown in Figures 8 and 9.

\subsection{Robustness of low-pass controllers with respect to shape uncertainties}

We have seen in Section 4.2 that if we slightly deform the optimal sensor of Figure 1, the modified system remains pseudo-collocated. As an example, if we replace the optimal sensor by the sensor drawn in Figure 10, the value of $\eta$ is 3.01 . 


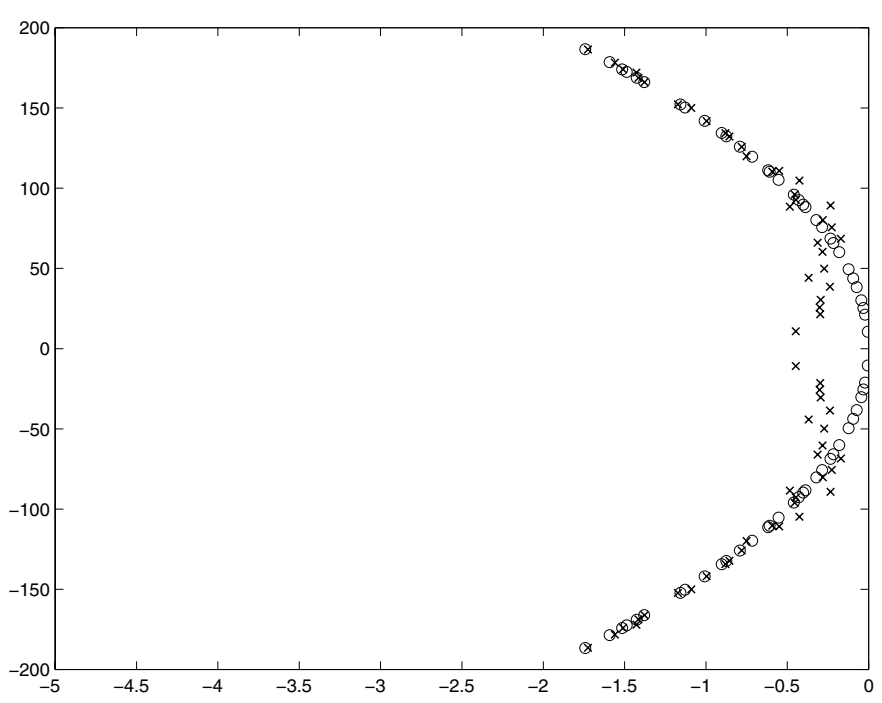

Figure 8. Poles of the open-loop (o) and closed-loop system (x).

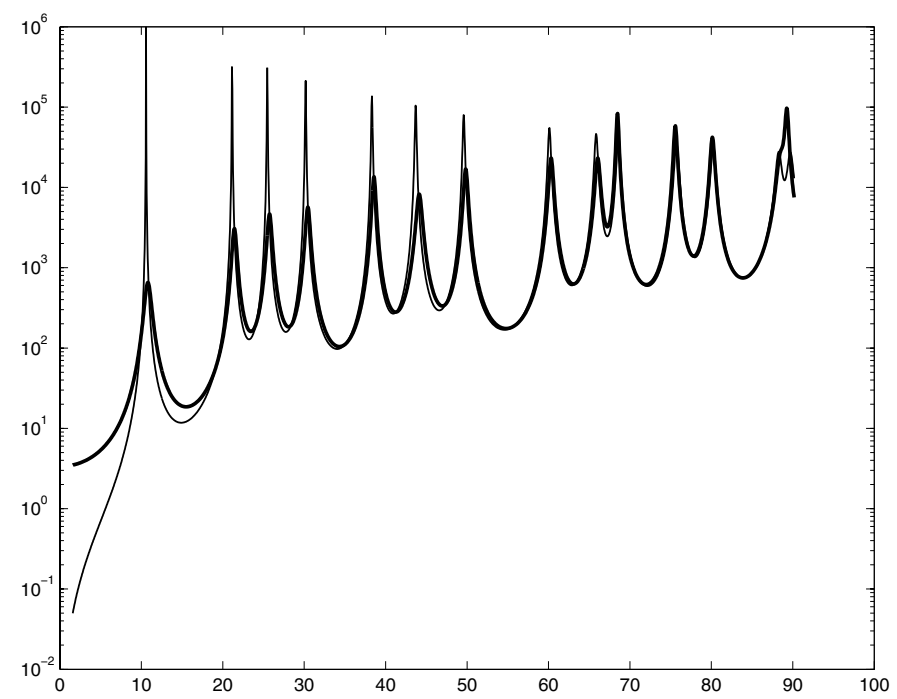

Figure 9. Magnitude of the mean energy with respect to the frequency of exogenous input, with (thick line) and without feedback (thin line).

If we denote this system by $G_{m}$, the following figures show that the controller $L$ computed in Section 5.2 also stabilizes the modified system $G_{m}$. This is due to the fact that the static gain $g_{0}$ has been chosen so that the eigenvalues of the closed-loop system are not too close to the imaginary axis.

\section{Concluding Remarks}

A new criterion for the design of non-collocated sensors and actuators has been performed. It consists in insuring the passivity of the system in the low-frequency domain. Our problem has been changed to a linear, infinite-dimensional, optimization problem, for which theoretical results have been obtained. First, the existence 


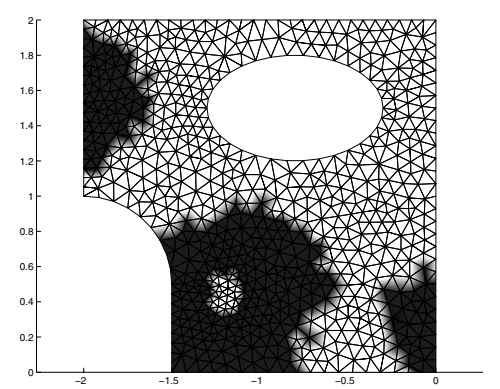

FiguRE 10. Deformation of the optimal sensor.

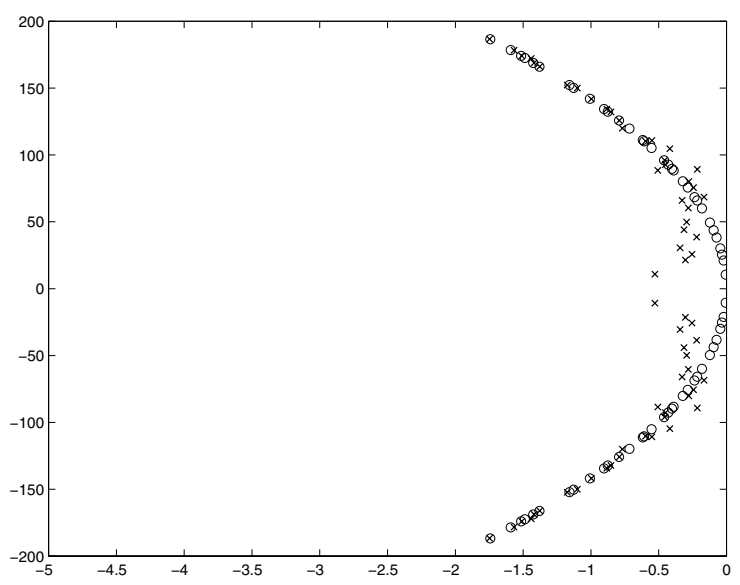

Figure 11. Poles of the open-loop (o) and closed-loop system (x).

of an optimal solution has been established. We have then shown that this solution is unique and is necessarily the characteristic function of a subdomain $\omega$ of the plate. This is an essential result in our study since a penalty term is useless and the optimization program remains linear. Although these results have been established for a particular system, they should hold for a more general class of well-posed second-order dissipative systems of the type

$$
z_{t t}+\mathcal{D} z_{t}+\mathcal{A} z=\mathcal{B} v
$$

with a damping operator $\mathcal{D}$, a scalar control $v$ and a scalar observation $y=\mathcal{C} z_{t}$ obtained through an operator $\mathcal{C}$ made of Dirac distributions or partial derivatives of Dirac distributions concentrated on the boundary of an open set $\omega \subset \Omega$. To this extent, the essential point would be the analyticity of the eigenfunctions of the operator

$$
A=\left(\begin{array}{cc}
0 & I \\
-\mathcal{A} & -\mathcal{D}
\end{array}\right)
$$

with domain $D(\mathcal{A}) \times D\left(\mathcal{A}^{\frac{1}{2}}\right)$. This needs, of course, a careful analysis. 


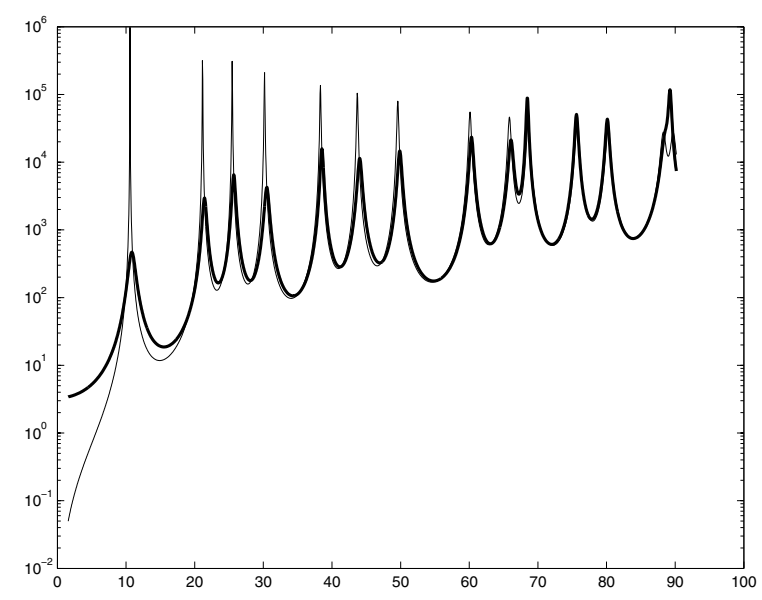

Figure 12. Magnitude of the mean energy with respect to the frequency of exogenous input, with (thick line) and without feedback (thin line).

Approximation of the optimal shape by $C^{0}$ piecewise linear finite elements has been computed for several meshes. It can be noticed that our method does not require the knowledge of the eigenmodes. The stabilization of the plate has also been studied: "low-pass" strictly positive real controllers have been used and the numerical results show the efficiency of this type of controllers.

The results obtained by topological optimization can be used to construct initial guesses for a classical shape optimization algorithm, thus circumventing the ill-conditioning. This coupling between both types of optimization has to be studied.

As we have pointed it in Section 3, the original criterion could be modified to take in account the behavior of the "uncontrolled" perturbation $\Delta_{K}$. The optimization problem could be modified as follows, with the notations of Section 2.2:

$$
\min _{f \in \mathcal{U}, \eta}-\eta
$$

under the constraints

$$
\begin{aligned}
\eta-w_{k} \mathcal{B}^{*} \varphi_{k} \mathcal{C} \varphi_{k} \leq 0, \quad k & =1 \ldots K \\
-\gamma \eta \leq w_{k} \mathcal{B}^{*} \varphi_{k} \mathcal{C} \varphi_{k} \leq \gamma \eta, & k>K
\end{aligned}
$$

where the weights $w_{k}>0$ are chosen such that $\left|\mathcal{B}^{*} \varphi_{k} \mathcal{C} \varphi_{k}\right| \rightarrow 0$ as $k \rightarrow \infty$, and $\gamma>0$ (a disjoint support or limited area constraint could be added as well). The idea is that this formulation still allows to maximize the response of the system in the control bandwidth (the first $K$ modes) while maintaining the response of "uncontrolled modes" small. It can be pointed out that the objective function and the constraints both remains linear. The smallest value of $\gamma$ such that (32)-(34) has a solution could be determined by a dichotomic search. The optimal $f$ obtained for this minimal value of $\gamma$ would give the best performance/robustness tradeoff.

Our last remark will be about the optimization of the pseudo-collocation criterion with respect to the shapes of the actuator and the sensor when both are of piezoelectric type. In this case, the problem we have to solve is

$$
\begin{gathered}
\min _{f, g \in \mathcal{U}, \eta}-\eta, \\
\eta-w_{k} \mathcal{B}^{*} \varphi_{k} \mathcal{C} \varphi_{k} \leq 0, \quad k=1 \ldots K
\end{gathered}
$$


where

$$
\mathcal{B}^{*} \varphi=\int_{\Omega} g \Delta \varphi, \quad \mathcal{C} \varphi=\int_{\Omega} f \Delta \varphi
$$

Numerically, (35)-(36) can be approximated by a linear program with indefinite quadratic constraints and usual algorithms such as Sequential Quadratic Programming cannot be applied. It can be noticed that (35) is linear when one of the variables $f$ or $g$ is fixed. An $a d$ hoc algorithm, which uses this bilinearity is currently being developed.

\section{REFERENCES}

[1] G. Allaire, Shape optimization by the homogenization method. Springer-Verlag, New York (2002).

[2] H.T. Banks, R.C. Smith and Y. Wang, Smart material structures, modelling, estimation and control. Res. Appl. Math. Masson, Paris (1996).

[3] D. Chenais and E. Zuazua, Finite Element Approximation on Elliptic Optimal Design. C.R. Acad. Sci. Paris Ser. I 338 729-734 (2004).

[4] M.J. Chen and C.A. Desoer, Necessary and sufficient conditions for robust stability of linear distributed feedback systems. Internat. J. Control 35 (1982) 255-267.

[5] R.F. Curtain and B. Van Keulen, Robust control with respect to coprime factors of infinite-dimensional positive real systems. IEEE Trans. Autom. Control 37 (1992) 868-871.

[6] R.F. Curtain and B. Van Keulen, Equivalence of input-output stability and exponential stability for infinite dimensional systems. J. Math. Syst. Theory 21 (1988) 19-48.

[7] R.F. Curtain, A synthesis of Time and Frequency domain methods for the control of infinite dimensional systems: a system theoretic approach, in Control and Estimation in Distributed Parameter Systems, H.T. Banks Ed. SIAM (1988) 171-224.

[8] R. Datko, Not all feedback stabilized hyperbolic systems are robust with respect to small time delays in their feedbacks. SIAM J. Control Optim. 26 (1988) 697-713.

[9] E. Degryse, Étude d'une nouvelle approche pour la conception de capteurs et d'actionneurs pour le contrôle des systèmes flexibles abstraits. Ph.D. Thesis, Université de Technologie de Compiègne, France (2002).

[10] P.H. Destuynder, I. Legrain, L. Castel and N. Richard, Theoretical, numerical and experimental discussion on the use of piezoelectric devices for control-structure interaction. Eur. J. Mech A/solids 11 (1992) 181-213.

[11] B.A. Francis, A Course in $H_{\infty}$ Control Theory. Lecture notes in control and information sciences. Springer-Verlag Berlin (1988).

[12] P. Freitas and E. Zuazua, Stability results for the wave equation with indefinite damping. J. Diff. Equations 132 (1996) 338-352.

[13] J.S. Freudenberg and P.D. Looze, Right half plane poles and zeros and design tradeoffs in feedback systems. IEEE Trans. Autom. Control 30 (1985) 555-565.

[14] J.S. Gibson and A. Adamian, Approximation theory for Linear-Quadratic-Gaussian control of flexible structures. SIAM J. Control Optim. 29 (1991) 1-37.

[15] A. Haraux, Systèmes dynamiques dissipatifs et applications. Masson, Paris (1990).

[16] P. Hébrard and A. Henrot, Optimal shape and position of the actuators for the stabilization of a string. Syst. Control Lett. 48 (2003) 199-209.

[17] P. Hébrard and A. Henrot, A spillover phenomenon in the optimal location of actuators. SIAM J. Control Optim., to appear.

[18] C. Inniss and T. Williams, Sensitivity of the zeros of flexible structures to sensor and actuator location. IEEE Trans. Autom. Control 45 (2000) 157-160.

[19] S. Jaffard, M. Tucsnak and E. Zuazua, Singular internal stabilization of the wave equation. J. Differential Equations 145 (1998) 184-215.

[20] T. Kato, Perturbation theory for linear operators. Springer-Verlag, Berlin (1980).

[21] B. van Keulen, $H_{\infty}$ control for distributed parameter systems: a state-space approach. Birkaüser, Boston (1993).

[22] I. Lasiecka and R. Triggiani, Non-dissipative boundary stabilization of the wave equation via boundary observation. J. Math. Pures Appl. 63 (1984) 59-80.

[23] D.G. Luenberger, Optimization by Vector Space Methods. John Wiley and Sons, New York (1969).

[24] F. Macia and E. Zuazua, On the lack of controllability of wave equations: a Gaussian beam approach. Asymptotic Analysis 32 (2002) 1-26. 
[25] M. Minoux, Programmation Mathématique: théorie et algorithmes, tome 2. Dunod, Paris (1983).

[26] O. Morgül, Dynamic boundary control of an Euler-Bernoulli beam. IEEE Trans. Autom. Control 37 (1992) 639-642.

[27] S. Mottelet, Controllability and stabilization of a canal with wave generators. SIAM J. Control Optim. 38 (2000) $711-735$.

[28] V.M. Popov, Hyperstability of Automatic Control Systems. Springer, New York (1973).

[29] F. Shimizu and S. Hara, A method of structure/control design Integration based on finite frequency conditions and its application to smart arm structure design, Proc. of SICE 2002, Osaka, (August 2002).

[30] V.A. Spector and H. Flashner, Sensitivity of structural models for non collocated control systems. Trans. ASME 111 (1989) 646-655.

[31] M. Tucsnak and S. Jaffard, Regularity of plate equations with control concentrated in interior curves. Proc. Roy. Soc. Edinburg A 127 (1997) 1005-1025.

[32] Y. Zhang, Solving Large-Scale Linear Programs by Interior-Point Methods Under the MATLAB Environment. Technical Report TR96-01, Department of Mathematics and Statistics, University of Maryland, Baltimore, MD (July 1995). 